\title{
Proceeding
}

Supplementary Issue: Spring Conferences of Sports Science. International Conference on Psychology of Education Sciences \& Lifestyle.

\section{Psychological and pedagogical technologies for the correction of social fears of participants in a subculture of role-playing games of live action}

\author{
ALLA V. FROLOVA \\ Department of Clinical Psychology and Psychology of Personality, Kazan (Volga Region) Federal University, Kazan, \\ Russian Federation
}

\begin{abstract}
Purpose of the study is to identify markers, "risk zones" that contribute to deviations among representatives of the subculture "roleviks", to elaborate a technology to correct negative experiences among "role" movement representatives to increase the efficiency of their adaptation. The following research methods were used in this study: "Questionnaire of the hierarchical structure of actual personal fears" (ISAS) methodology to identify phobias; Personality Belief Questionnaire - PBQ to specify dysfunctional beliefs impeding adaptation (emotional well-being, constructive relationships with other people, etc.). The research results suggest that a systematic integrated approach is a necessary condition for the success of the psychological technology aimed at overcoming negative experiences and maladaptation of "role" movement subculture representatives. A combination of diagnostic and correctional models to overcome social fears, deprivation and maladaptation makes up the essence of the technology. The research provides findings that can be used by managers of various levels in the education system, social workers, teachers and practical psychologists in the education system; they can also be of use while organizing special programs for educators, social and psychological services. It has been worked out to help students - "roleviks" to overcome their social fears. It represents a system of single interrelated activities determined to form and develop personal adaptive resources. It has been established that positive changes in adaptation strategies, a decrease in the level of social fears and anxiety are the main criteria for evaluating the effectiveness of the presented psychological-pedagogical technology. It is shown that the applied technology promotes the decrease of experienced fears intensity, forms stress resistance, and contributes to the selection of effective social interaction strategies.
\end{abstract}

Keywords: Youth subculture of role-playing games; Fears; Sociophobia; Deviation; Technologies; Adaptation.

Cite this article as:

Frolova, A.V. (2020). Psychological and pedagogical technologies for the correction of social fears of participants in a subculture of role-playing games of live action. Journal of Human Sport and Exercise, 15(3proc), S879S888. doi:https://doi.org/10.14198/ihse.2020.15.Proc3.39

Corresponding author. Department of Clinical Psychology and Psychology of Personality, Kazan (Volga Region) Federal University, Kazan, Russian Federation.

E-mail: alfrol1@mail.ru

Supplementary Issue: Spring Conferences of Sports Science. International Conference on Psychology of Education Sciences and Lifestyle.

JOURNAL OF HUMAN SPORT \& EXERCISE ISSN 1988-5202

(c) Faculty of Education. University of Alicante

doi:10.14198/jhse.2020.15.Proc3.39 


\section{INTRODUCTION}

\section{Relevance of the problem}

Currently, the issue of youth subculture has moved from the socio-cultural to the pedagogical field of research; scientists emphasize the importance of studying the specificity of youth subcultures (Austin, 2008) due to the fact that they are present in modern society and manifest themselves in various degrees and forms, thus exerting a significant impact on young people' education: the emergence of new cultural and value orientations in the educational space are often associated with destructive and nihilistic tendencies in the sphere of personal identity (Daniau, 2016; Martsinkovskaya, 2014; Poleva, 2018). The problems of culture and youth subculture interaction, the transition of cultural values and formation of new guidelines in the society are relevant (Salen \& Zimmerman, 2004; Schechner,2006). Understanding the role of subculture, its influence on the formation of spiritual and moral values substantiate the emergence of new requirements for the teacher and psychologist and should be regarded when educational and preventive programs are elaborated in an educational institution.

It is argued that, on the one hand, subcultures have an impact on the culture in the society, they become a way of self-realization and one's own position formation, often alternative to the public one; they act as a form of creative potential expression, as a way of building own world; on the other hand, they have the status of "externality" which creates difficulties to interact harmoniously with the surrounding reality (Kaplan, 2002). Subcultural identity is often constructed and functions in unofficial (including gaming) forms. Every cultural form can be considered as a game, and the essence of the youth subculture is especially revealed through the game (Carloson, 2004; Heyzinga, 2017); the game is one of the five main phenomena of human being. Thus, the leading priority of the role-playing subculture is the game as a transformed being. The specificity of the role-playing movement causes a non-random interest, since a psychological analysis of its social role and forms of response that representatives of this movement give are of great importance for understanding an individual social behaviour. The scientific community suggests that the role-playing culture is a way of social escapism connected with intense anxiety and fear of evaluation, distress and maladaptation of the individual (Shcherbachev, 2014). In this regard, the issue of studying the role-playing subculture representatives in the context of youth socialization opportunities is relevant.

\section{Literature analysis}

Subculture is understood as the culture of a certain group or community that is different from the one dominating in the society. One of the most common options is the youth subculture, this or that movement of adolescents and young people manifesting norms and cultural peculiarities that differ from the standards generally accepted in society.

As for the present-day situation, the phenomenon of youth subculture has been widely studied by foreign researchers. The phenomenon of youth subculture takes its roots in the second half of the 20th century. The United States of America is considered the country of origin (Bentley, 2005). Scientists define the peculiarity of subculture development dynamics as a mixture, when one modern style partially "covers" the other. Thus, there is such a tendency of subculture development as a mixture of subcultural styles. In an attempt to explain the mixture of styles, scientists resort to the term "habitat" ("cultic milieu"), which serves as a unifying foundation for representatives of new subcultures (Kaplan, 2002). Within the "environment", subcultures strive to divide into more specific areas, as well as to bring ideas and values closer together. For example, straight edgers, having purely individual characteristics, share common values with environmentalists and fighters with sexual licentiousness. According to the researcher, the fact that many directions appear in this 
or that subculture simply excludes the possibility of their further autonomy. This leads to the situation that over time they begin to contact and gradually borrow ideas and values from each other, losing their specificity.

Nowadays, three main approaches to the study of youth subculture are identified: structural functionalism approach, class and post-subcultural approaches. When studying this problem, foreign researchers consider the youth subculture through the paradigm of concepts of deviant behaviour, delinquency and socialization (Cohen, 1955) of youth subcultures. This typology takes into account psychological characteristics of the individual. The structural functionalism approach explained the problem of youth crime groups spread in rapidly growing cities. Representatives of this approach for the first time proposed an understanding of "delinquency" as a more or less "normal" reaction to the social environment, as opposed to "a manifestation of psychological deviations". Empirical data acquired particular importance: communication and observation of people's interactions when revealing the social way of their life. In order to understand why a group of young people got involved in illegal activities, scientists tried to analyse the external social environment of young people with possible perspectives or lack thereof. For example, in the case of the criminal group "delinquent boys" (Cohen, 1955), it was shown that their behaviour was the reflection of such structural problems as the organization of education, employment, and not as a consequence of their natural "abnormality". With regard to the social context, it was assumed that deviant behaviour is the product of "labelling": social groups cause deviation by creating rules, and those who violate them are labelled as outsiders (Beck et al., 2001). As a result, delinquent subcultures find themselves trapped: the more they repulse the dominant society, the greater the scale of social stigma.

According to the structural functionalism approach, society is regarded as a biological system and represents a "living organism". The constituent elements of the society are the family, education, economics, social institutions which, being closely connected, form a "functional whole", thereby ensuring a social order (Clark,2003). Pilkington was the first to apply this approach; according to the researcher, the main function of subculture was to mitigate the transition of young people into adult status (Pilkington, 1994). The significance of young people's socialization was emphasized; it was pointed out that they should be socialized before they attained adult status; moreover, the importance of getting education for the implementation of adult roles was specified. As specified by Pilkington the youth subculture represented the period of young people's preparation for life outside the family.

Therefore, subculture is a kind of "an adaptive form" that helps the whole society to achieve stability. Merton kept to a different view on social stability. He believed that to make the society function properly, people should be able to achieve those goals that the society established for them (Merton, 1957). If they fail to do this, they begin to experience psychological discomfort and, as a consequence, they can cast doubt on the legitimacy of social rules and jointly create other alternative values and means to achieve these goals, thereby forming subcultures. If a young person cannot achieve respect and status through traditional means, then unconventional, often illegal means, will come to the rescue. Criminal subculture participants, who accept alternative values, often admit deviant behaviour (deviating from generally accepted norms) as acceptable and magnify it.

Sociologists-functionalists give definition to subculture and emphasize that subculture is an element of culture, but at the same time it is different from the latter due to its own specific character within the general culture of society. It can be assumed that subcultures are derivatives of culture that form necessary degrees of freedom within the framework of the general system. 
One of the most common youth informal movements is the role-playing movement. The system-forming element of the role-playing movement is role-playing games. Their classification can be based on various features: the place and time of the game, the nature of the game genre, the degree of symbol usage, and the level of virtuality. The phenomenon of role-playing games is international in nature; there are international traditions of researching role-playing games of live action - live role playing games (Irpg), or live-action roleplaying (larp). Works of some foreign researchers examine ritual practices of the role-playing subculture (Alexander, 2004; Balme,2008; Müller, 2011).

Live role-playing games represent role-playing games remotely related to the theatre or psychodrama, as well as business games. Another difference is that the game has a very large element of real action: chases, battles, travels. A role-playing game is a game whose participants for some time live the life of any characters in a specially organized, artificially created world. They can be characters of a novel, a fantastic story, heroes of a real story or even creatures created by the players themselves. D.B. Pisarevskaya (2011) conducted the most complete and systematic study of the role-playing community. This anthropological study examined directions of socialization in role-playing movement, the dialogue between representatives of the subculture from different countries, as well as the dialogue between the subculture of role-playing games and basic culture; ritualized practices and folklore of the role-playing movement were also described (Pisarevskaya, 2011).

A distinctive feature of this subculture is that role-players are an informal group, there is no clearly defined hierarchy of the type "there is only one leader - all others are members of the group"; there is no such thing as "a leader" and "an outsider". The following role differentiations can be distinguished inside the subculture: the master (the person who invents and plays the game) and the player (a game participant), as well as the older and younger generations of role players. (Björn-Ole, 2011; Bennette \& Kahn-Harris, 2004).

One more characteristic feature of this movement is the need to reduce their socio-psychological age. This feature can be interpreted as a form of infantilism, a demonstration of regressive behaviour, as a return to childish forms of behaviour and thinking, as a "stuck" in the early stages of development which can lead to social maladaptation, difficulty in interaction with the surrounding reality and, as a result, provoke the appearance of addictive behaviour.

\section{Purpose of the study}

The purpose of the research is to study features of negative experiences, to identify markers, "risk zones" that contribute to the formation of deviations among representatives of the "role-playing" subculture; to develop a technology aimed at correcting negative experiences among representatives of the "role-playing" movement as a tool to enhance the efficiency of their adaptation.

\section{METHODS}

The following methods were applied in the work: theoretical and participant observation methods, an unsystematic continuous observation method was also chosen in order to collect the maximum amount of information to be aware of what was happening in the observed group. Participants' appearance, their statements in a free form during a natural conversation, materials of their pages on the social network "Vkontakte" made up the basis of the assessment.

The diagnostic tools: "The questionnaire of the hierarchical structure of actual personal fears" (HSAF) by included Y. Shcherbatykh and E. Ivleva (1998); methodology for identifying phobias; a questionnaire of 
personal beliefs (The Personality Belief Questionnaire - PBQ, A.T. Beck et al. (2001)) to identify dysfunctional beliefs that impede adaptation (emotional well-being, establishment of constructive relationships with other people, etc.). The "Coping Test" by R.S. Lazarus and S. Folkman (1984) was used as an additional research method.

Methods of mathematical statistics were applied to process obtained empirical data. Statistical data processing was carried out using the software packages 'STATISTIKA 6.0" and "SPSS 13.0".

\section{Research experimental base}

The research was carried out at higher educational institutions in the city of Kazan. The empirical basis of the study was the diagnostic results of 100 people: university students, males and females who belonged and did not belong to the "role-playing" subculture. The participants were aged between 17-23 years.

\section{Experiment stages}

In the first stage of the research, the participants were divided into two groups: the first group consisted of 50 participants of the "role-playing movement", the second group included 50 students who did not belong to the "role-playing movement"; they were all diagnosed to identify negative experiences, namely, fears, phobias, dysfunctional beliefs that impede adaptation; the risk of distress experiences was assessed among "role-playing" students with the help of a complex phased-based psychological diagnostics aimed at determining negative factors in groups of "depressive" risk. We elaborated a psycho-diagnostic package in compliance with our goals which was based on the following criteria: establishing the level of social phobia experience, distress, manifestation of depression features, preferred adaptation strategy, and identifying "risk" groups.

In the second stage of the research, in accordance with the data obtained, a training program compiled with regard to the specificity and depth of experienced negative forms of fear, the level of distress, as well as features and problems of participants in the "role-playing" movement as a social group was implemented. Psychological training is designed to form cognitive skills and abilities, positive identity, and psychological stability.

\section{RESULTS AND DISCUSSION}

The worked out technology for correcting social fears among "role-playing" university students includes two blocks: diagnostic and psycho-correctional. In the first stage, the so-called risk groups were identified in the group of respondents, namely, persons with the greatest deviations from the norm in compliance with the following indicators: manifestation of certain fears in interconnection with dysfunctional attitudes and coping. "The Questionnaire for Identifying Actual Fears" made it possible to analyse the main groups of existential fears: fear of the future and unknown life. The following fears were relevant for those respondents who did not belong to the "role-playing movement": death, old age, poverty, future changes in their personal lives. The ranking showed that fear of death occupied the fifth position in the group of respondents who did not belong to the "role-playing movement", and the tenth in the group of respondents - "role-players". The comparison of actual fears manifestation values in the groups of respondents was conducted using the Student t-test; it allowed us to conclude that at significance levels $(p \leq .01)$ or $(p \leq .05)$ there were differences in three values: fear of the future ( $t_{\mathrm{emp}}=2.643$ ), fear of the negative consequences of diseases in loved ones $\left(t_{\text {emp }}=-2.319\right)$, fear of contracting any disease (temp $\left.=-1.995\right)$. When anxiety is defined as a person's reaction to threatening situations, it is important to understand which situations respondents assess as anxious. The research revealed that the level of social fears in the group of students from the role-playing subculture was 
higher on almost all scales. High rates for this technique indicate the manifestation of internal insecurity, tension, irrational fear of performing any social actions and actions accompanied by attention from outsiders. The main component of these fears is the fear of being negatively assessed by others. In the group of students who were not members of the "role-playing movement" subculture, indicators on the level of social fears were moderate; it testifies to the level of their adaptability and ability to overcome anxiety. As it was noted, the fear of exams (average value is 6.23) and the fear of responsibility (average value is 6), as well as the fear of public speaking were most pronounced in the group of "role-players" respondents. High indicators on the subscale of the fear of responsibility indicate the presence of social evaluative fear; it is obvious that the fear of responsibility is associated with the experience of shame, fantasies about condemnation and the emergence of self-blaming reactions. The results obtained in the group of role-players respondents may indicate social avoidance, and such a specific way of interacting with the surrounding reality as "escaping" to the world of fantasies and illusions. The results show that, in general, the level of personal anxiety affects the intensity of manifestation of fear, as evidenced by a high significant correlation $(r=.489$ at $p=<.01$ ). An important task in this study was to determine the relationship with fear coping strategies.

The highest indicator of the preferred mechanisms of coping behaviour was obtained on the subscale "escape-avoidance", this coping strategy was preferred by $77 \%$ of respondents with an average value of 7.17 points. The second place was taken by the "positive revaluation" coping strategy typical for $17 \%$ of roleplaying movement respondents, with an average value of 6.29 points, and $16 \%$ of respondents from the roleplaying group coping strategy were characterized by a distancing strategy, with an average value of 4, 26 points. Other strategies were not preferred ones. The choice of these strategies reflects the desire and behavioural efforts aimed at escaping or avoiding the problem, using passive strategies aimed not at solving the problem, but at reducing emotional stress. It can be assumed that the choice of the "Positive revaluation" strategy is associated with the mechanism of idealization, the purpose of which is to remove anxiety and possible confrontation when meeting an object that causes fear. Representatives of this group prefer not to pay attention to their fears (dislodge); thus, they use the "positive revaluation" mechanism as strategies for coping with fear.

The correlation analysis revealed an inverse correlation relationship (-.53) between the two coping strategies "Confrontation" and "Avoidance" in the group of role-playing respondents.

Spearman's correlation analysis specified statistically significant relationships (at $p<.01^{* *}$ and at $p<.05^{*}$ ) between certain types of fear and dysfunctional beliefs. So, for example, in the group of role-playing respondents the fear of public speaking significantly correlated with beliefs: "It is important to do any job perfectly", "I have to do everything in my own way"; respondents with agressophobia (fear of physical aggression: before an attack, a fight), fear of mental aggression (screaming, rudeness, humiliation), fear of being looked at, dominant fear of bosses and higher-ranking people adhered to the statements "I have to completely control emotions", "It's better not to express your anger directly, but to show displeasure by disobedience"; respondents from role-playing movement with addictive fears (fear of lack or absence of an addiction agent: food, money, tobacco, alcohol, drug, person) were associated with the statement "In my essence, I am unacceptable"; fear of exams is accompanied with the following statement "The world is dangerous and hostile", "I am powerless and vulnerable". Expressed indicators were obtained in the sample of role-player respondents on the subscales "addiction" - "defence"; the following statements are typical for them: "If people get to know me better, they will understand who I really am and reject me"); it is connected with the experience of low value and shame, and reflects own opinion of oneself as unworthy of a good attitude from others; "Unpleasant feelings will grow and go out of control" - the belief characterizes the feeling of vulnerability, fear of the inability to control your negative emotions, the desire to present yourself in a better 
light. The perception of other people as "unkind and indifferent" can generate the emergence of experiences connected with lowering mood, namely: alertness, suspiciousness, resentment; it may stimulate conflict, distant relationships. Such experiences can determine maladaptation and be a source of distress. The belief about the "hostility", "inaccessibility" of the surrounding world actually reflects the lack of perceived social support which, in turn, is the main factor of successful stress management, and is one of the factors for successful adaptation. The nature of correlations with the parameters of basic personality settings suggests that evaluative anxiety, to a greater extent than other types of anxiety, is associated with the "defectiveness" of the basic settings regarding the self-image (non-acceptance of oneself, fear of knowing oneself, distrust of oneself, unwillingness and inability to accept changes taking place in the implicit self-concept), and to a lesser extent, with basic attitudes regarding the surrounding world.

The research specified that the groups of respondents significantly differ as for the indicator which we conditionally define as "interpersonal sensitivity" and which is characterized by a "feeling of personal inadequacy and inferiority", negative expectations regarding social interaction, anxiety and discomfort in the process of social interactions, which makes up the core of the social phobia phenomenon. Emotional vulnerability, hypersensitivity to adverse stimuli, a high level of anxiety are the main characteristics of the emotional sphere of "role-playing" respondents.

In was found that students - "role-players" use the "flee-avoidance" defence mechanism as strategies for coping with fear. Probably, the impossibility to overcome the fear of social contacts is supplanted by the fact that "role-playing" students go there where they can realize themselves by switching to another activity (playing), due to which they maintain a sense of control over reality. Thus, they are able to reduce their experience of fear through specific physical or mental forms of activity.

It should be noted that in their defensive behaviour, respondents rely on the picture of the world that is most characteristic for them, e.g. respondents sticking to the belief that "the world is dangerous and hostile" use such a strategy of coping behaviour as distancing.

Then, in the second stage, after analysing obtained diagnostic data, correctional training aimed at recognizing the attributive style was carried out; it enabled us to understand and change the usual way of thinking about the causes of events which determine the degree of belief in success and the degree of persons' persistence in difficult situations, the formation of assertive behaviour skills, overcoming of negative thoughts, and analysis of own expectations. Following the cognitive-behavioural training, diagnostics was carried out to verify the effectiveness of this program. When defining risk "zones", the following criteria were important indicators for overcoming stressful situations: cognitive criterion for self-understanding, self-relationship (with indicators of positive emotions and motives); behavioural criterion for self-development (with indicators of a successful behaviour strategy to affect stressful situations); reflexive criterion for self-improvement (with indicators of factors of stressful reactions anticipation). Based on the above criteria, we proposed a correctional model to manage negative emotions (anxiety and social fears).

Data analysis of the program effectiveness testifies in favour of the fact that a statistically significant decrease in social fears was observed after the training. For example, a comparison of the values of actual fears manifestation, according to the results of questionnaires, before and after the training, also indicates a significant difference in the fear index according to the following parameters: illnesses of loved ones (2.37), fear of death (1.20), fear of changes in personal life (1.15), fear of old age (0.70), fear of: negative consequences of diseases of loved ones (0.60), fear of getting sick (0.42), fear of the future (0.38). It was revealed that the fear of poverty remained within the framework of previous indicators. The comparison of 
the levels of actual fears manifestations in the group of role-playing respondents provided significant differences in the manifestation of fear of death $(p<.01)$.

Our research results are consistent with the data from other works and point out problems of youth subculture representatives' adaptation, as well as difficulties they face while choosing coping strategies.

With regard to researchers' points of view, we assume that the main function of the youth subculture is to provide opportunities for self-identification and self-realization; the subculture often acts as an important coping mechanism applied to overcome social fears. The results obtained correspond to Brake's concept (), who argues that subculture performs the most important functions: provides a symbolic solution to problems and contradictions that are collectively experienced by the generation; offers values, ideology and lifestyle that contribute to the formation of identity outside the prescribed, given solidarity; represents an alternative form of social reality.

The research confirms the view that the opportunity of active confirmation of one's self and self-presentation reduces the role of the struggle of identities and, partially, escape. At the same time, role-playing subculture, on the one hand, enables flexible and positive socialization, on the other hand, it offers options for a "game" with one's own identity, i.e. chances to try yourself in different masks and different roles to increase the awareness of both the role repertoire and own performance of different roles (Martsinkovskaya, 2014).

We have shown that a specific feature of the role-playing movement participants was the assessment of the world around them as "indifferent and "unkind". V.V. Shcherbachev (2014) in his study asserts that the most important psychological trait of the role-playing movement participants is an internal conflict between the need for recognition and admiration and perception of the environment as hostile. The author suggests that role-playing facilitates the avoidance from reality, on the one hand, and, on the other hand, makes it possible to adapt to the environment through playing social situations (Shcherbachev, 2014).

Thus, all researchers agree that the role-playing movement in one way or another has adaptive and socializing functions. At the same time, authors pay little attention to the issue how the change of images and roles affects the overcoming of negative experiences and determines the specificity of socialization.

\section{CONCLUSION}

The study allows us to draw the following conclusions.

It was established that groups of participants have a different structure of experienced fears.

It was found that groups of respondents significantly differ on the indicator characterized by negative expectations regarding social interaction, anxiety and discomfort in the process of social interactions, which makes up the core of the phenomenon of social phobia. Emotional vulnerability, hypersensitivity to adverse incentives are main characteristics of role-playing respondents' emotional sphere. From our point of view, this reflects distancing, their fear of social contacts. Respondents who do not belong to the role-playing movement are significantly more satisfied with social support than role-players respondents.

It was shown that the emergence of social fears of role-players respondents is based on interpersonal factors: tendency to idealize people around them, high expectations of a "good attitude" from others, on the one hand; 
on the other hand, distrust of people, hostility, inconsistency of affection and tendency to breakdown relations when even the slightest problems appear.

It was established that the role-playing subculture is an important coping mechanism for overcoming social fears. Role-playing is a certain hyper compensation that allows one to manage own inferiority through playing different role-playing images.

Conditions for overcoming social fears of role-playing movement participants are as follows: formation of emotional decentration, decrease in aggressiveness, development of self-esteem, and provision of adequate interpersonal interaction with others. An effective means of teaching to overcome offence and resentment is the socio-psychological training of an integrative type, each direction of which is practiced at three levels: cognitive, emotional and behavioural.

The elaborated model of psychological and pedagogical correction of fears includes emotional and value components that ensure the formation of knowledge, skills and attitudes to existential fears. As a result, students acquire knowledge about their own fears, causes of their occurrence and mechanisms of appearance; skills of constructive behaviour, assessment of traumatic situations, reflection of traumatic situations, regulation of emotional states; value attitude to overcoming of emerging fears and anxieties.

The application of the targeted program of psychological and pedagogical correction of fears among respondents of role-playing movements revealed a decrease in the level of anxiety, manifestation of actual fears, in particular, existential fear of death and socially evaluative fear, as one of the most significant fears.

\section{ACKNOWLEDGEMENTS}

The work is performed according to the Russian Government Program of Competitive Growth of Kazan Federal University.

\section{REFERENCES}

Alexander, J.C. (2004). Cultural Pragmatics: Social Performance between Ritual and Strategy. Sociological Theory, 22(4), 527-573. https://doi.org/10.1111/j.0735-2751.2004.00233.x

Austin, J. (2008). Youth Culture. Encyclopedia of Children and Childhood in History and Society Wh-Z.

Balme, C. (2008). The Cambridge Introduction to Theatre Studies. Cambridge: Cambridge University Press.

Beck, A.T., Butler, A.C., Brown, G.K., Dahlsgaard, K.K., Newman, C.F. \& Beck, J.S. (2001). Dysfunctional beliefs discriminate personality disorders. Behaviour Research and Therapy, 39, 12131225. https://doi.org/10.1016/s0005-7967(00)00099-1

Bennette, A. \& Kahn-Harris, K. (2004). After Subculture. Critical Studies in Contemporary Youth Culture. New York: Palgrave Macmillan.

Bentley, N. (2005). The Young ones: a reassessment of the British New Left's representation of 1950s youth subcultures. European Journal of Cultural Studies, 8, 65-82. https://doi.org/10.1177/1367549405049492

Björn-Ole, K. (2011). Why Japan does not Larp. Think Larp - Academic Writings from KP2011, First Edition. https://www.academia.edu/3462882/Why_Japan_does_not_Larp

Carlson, M. (2004). Performance: a critical introduction. New York: Routledge. 
Clark, D. (2003). The Death and Life of Punk, the Last Subculture. The Post-Subcultures Reader, David Muggleton \& Rupert Weinzier (Eds.). Oxford: Berg, 223-236.

Cohen, A.K. (1955). Delinquent Boys: The Culture of the Gang. Glencoe: The Free Press.

Daniau, S. (2016). The Transformative Potential of Role-Playing Games: From Play Skills to Human skills. Simulation \& Gaming, 47(4), 423-444. https://doi.org/10.1177/1046878116650765

Heyzinga, J. (2017). The man playing: experience of determining the game element of culture. Moscow: Ivan Limbach Publishing House.

Kaplan, J. (2002). The Cultic Milieu: Oppositional Subcultures in an Age of Globalization. Walnut Creek: Alta Mira Press.

Lazarus R.S. \& Folkman S. (1984). Stress, appraisal and coping. New York: Springer Publishing Company.

Martsinkovskaya, T.D. (2014). Psychology of subculture. Bulletin of the Russian Humanitarian Scientific Foundation, 4, 114-124.

Müller, M. (2011). From Subculture to Mainstream. Think Larp - Academic Writings from KP2011, First Edition, 76. Copenhagen: Rollespilsakademiet.

Pilkington, H. (1994). Russia's Youth and its Culture. A Nation's Constructors and Constructed. LondonNew York: Routledge. https://doi.org/10.4324/9780203421116

Pisarevskaya, D.B. (2011). Subculture of role-playing games in modern society. Moscow: IEA RAS.

Poleva, N.S. (2018). From identification to identity. Psychological research, 58(11), 3-16.

Salen, K. \& Zimmerman, E. (2004). Rules of play: game design fundamentals. Cambridge: Mass: The MIT press.

Schechner, R. (2006). Performance Studies: An introduction. London: Routledge.

Shcherbachev, V.V. (2014). Individual psychological characteristics of participants in a subculture of roleplaying games of live action. Yaroslavl Pedagogical Bulletin (Psychological and Pedagogical Sciences), 3(2), 217-221.

Shcherbatykh, Yu.V. \& Ivleva, E.I. (1998). Psikhofiziologicheskie i klinicheskie aspekty strakha, trevogi i fobii. Voronezh: Istoki. 